\title{
PAPER \\ Optical Wireless Communications and Autonomous Beam Control Moving User Terminal
}

\author{
Charoen TANGTRONGBENCHASIL ${ }^{\dagger a)}$, Yoichi HAMADA ${ }^{\dagger}$, Nonmembers, Toshihiro KATO $^{\dagger}$, Student Member, \\ and Koji NONAKA ${ }^{\dagger}$, Member
}

\begin{abstract}
SUMMARY Optical wireless communications is a research topic of extreme interest since it offers high data rate (Gbps data rate), security, and RF interference immunity. However, optical wireless communications places severe restrictions on the communications paths; they must be direct beam connections. To increase the number of users and link robustness, optical wireless communications must be able to operate even when obstacles are placed between transmitters and receivers, so optical micro-cell (OMC) with autonomous beam control can overcome link robustness. In addition, OMC based optical wireless communication yields compact systems. This paper presents the design, an implementation, and a demonstration of a 114 Mbps autonomous beam control optical wireless communication system based on an OMC technique. The robust posture control results optimum downlink alignment and good eye diagram of data transmission.

key words: optical wireless communication, optical micro-cell system, $\mathrm{VC}$ SEL array, APD array, beam control
\end{abstract}

\section{Introduction}

The mobility of sound and visual communications has become a key technology of communication. Present commercial available wireless communication carrier is radio frequency (RF) wave providing mobility over large coverage areas. However, RF is limited by radiation bandwidth and carrier transmission due to radio law regulation. At present, RF wireless communications standard or IEEE $802.11 \mathrm{G}$ provides only $54 \mathrm{Mbps}$ total data rate [1]. In addition, RF devices disturb autonomous systems and medical instruments. Therefore, optical wireless communication is proposed to overcome bandwidth and RF interference problems due to very high carrier frequency bandwidth and no RF interference. When communication medium is photonic wave, it provides much higher speed modulation due to the very high carrier frequency bandwidth approximately $300 \mathrm{THz}$ at wavelength of $1 \mu \mathrm{m}$ [2]. Moreover, the wavelength multiplexing technique can provide a very large capacity and multi-user accessibilities. In addition, optical radiation does not disturb electromagnetic sensitive appliances in the same service area.

The different kinds of link for indoor optical wireless communications have been classified, depending on the existence of a line-of-sight (LOS) path between the transmitter $(\mathrm{Tx})$ and receiver $(\mathrm{Rx})$, and the degree of directionality

\footnotetext{
Manuscript received December 21, 2006.

Manuscript revised May 21, 2007.

${ }^{\dagger}$ The authors are with Kochi University of Technology, Kamishi, 782-8502 Japan.

a) E-mail: 096401e@gs.kochi-tech.ac.jp

DOI: 10.1093/ietcom/e90-b.11.3224
}

(directed, distributed, or hybrid) [3]-[6]. Directed links improve power efficiency as minized path loss but this kind of systems strictly requires alignment technique for both of Tx and Rx. It makes systems less convenience for practical applications. One of most attractive configurations is the distributed system. Operation under this configuration does not require a direct LOS or strictly alignment between Tx and Rx because the photonic waves are spread as uniform distribution [5], [6] making system to be the most robust and flexible configuration. However, distributed system suffers power detection problem due to eye-safe power density, coverage distance, and signal bit rate. To compensate both directed and distributed links problems for high speed optical wireless communication, a combination of two solution concepts is proposed; one is optical micro-cell (OMC) hub node [7]-[10] and another is high sensitive user terminal which features optical Rx such as avalanche photodiode (APD) with autonomous lens posture beam control [10][13] for non-directed links with improved receiving power at user terminal. Ref. [10]-[13] implemented 5-channel receiver array which is high optimization for beam detection and signal communication but cost is still high. The commercial available 4-channel APD receiver array developing for beacon usage was capable for implementation of the similar characteristic to detect beam direction and signal communication and cost consequently is reduced.

\section{Overall System}

The concept of hub node is named as OMC system which is shown in Fig. 1 [7]-[10]. It consists of an optoelectronic array hub node and user terminals. Hub node consists of a vertical cavity surface emitting laser diode (VCSEL) array and an photodiode (PD) array acting as transmitter array and receiver array, respectively. Electronic switching matrixes are required for selecting the proper communication channels (VCSEL and PD cells) for multi-user accessibility. Hub node also requires signal processing modules amplifying and filtering the detected optical signal. The signal processing modules also control the electronic switching matrixes for selecting the proper communication paths.

At user terminal, a VCSEL and a 4-channel APD were implemented as transmitter and receiver, respectively. Signal from 4-channel APD were also processed by signal processing module amplifying, filtering, and controlling user terminal posture. The user terminal signals contain quasi- 
DC signal and AC signal using for beam control and data communication, respectively.

Figure 2 shows the overall optoelectronics, signal processing \& control circuit, and interface module block diagram. This system can operate not only Ethernet but also

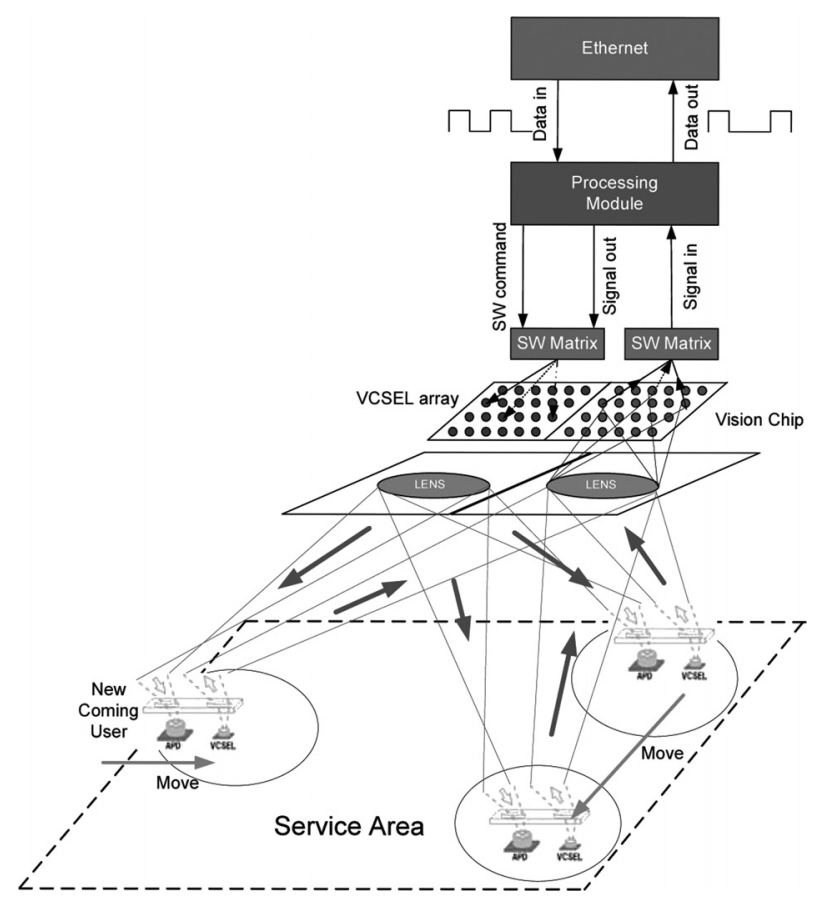

Fig. 1 Optical micro-cell system. overhead camera (OHC) projector and other communication devices, even though the interfaced protocol is changed. While user terminal were moving into a service area of a hub node, 4-channel APD with a single lens of user terminal detected communication light intensity, and then posture was automatically adjusted. Consequently, the received signals were filtered and amplified by signal conditioner module (S.C.) to adjust to be proper voltage level and frequency band for further signal processing. The filtered and amplified signals were also transmitted to user terminal's controller to decide and then user terminal's controller sent a control command to adjust lens posture to achieve balanced optical signal condition. When balanced optical signal condition were realized, user terminal's controller then transmitted a command to initiate link with hub node by media access control (MAC) address and initialized command to hub node via user terminal VCSEL. When hub node received MAC address and initialized command, hub node's controller acknowledged user terminal and waited for next commands for next communications.

However, when other users move into the service area of hub node, electronic switching matrix is additionally required to switch data to different cells or service areas of VCSEL array in the same hub node. In this situation, control command (e.g. cell number and/or handover requirement) was required to insert in transmitting signal both of hub node and user terminal. On the other hand, the control command of a mobile system is not necessary wideband. Sub-Mbps control command is sufficient for real-time cell switching and user terminal autonomous posture controls. In order to

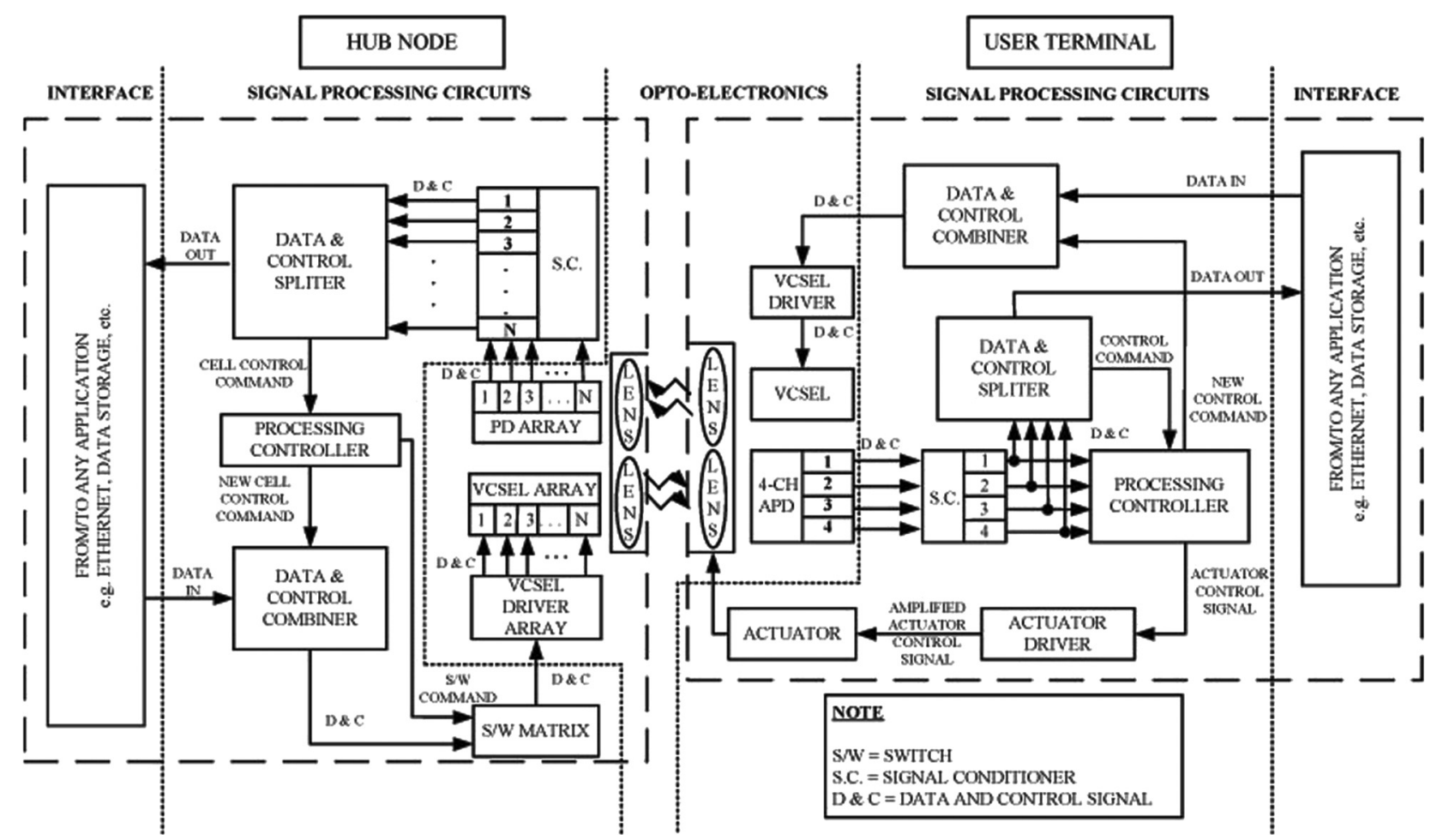

Fig. 2 System block diagram both of hub node and user terminal. 
combine control command (low speed signal; sub kbps) and data signal (high speed signal; faster than $100 \mathrm{Mbps}$ ) in a single frame communication, phase-locked loop (PLL) circuits were adopted as a frequency synthesizer to generate a clock signal for the data buffer in Tx and a clock recovery system in Rx to retrieve the clock [14].

\section{Optoelectronic Configuration}

By the advanced integration technology in optoelectronics, VCSEL array based optical communication has great potential of total capacity up to 120 Gbps [15]-[18]. Array size of hub node is only a few $\mathrm{mm}^{2}$, so, VCSEL array with a single lens was implemented as a compact multi-Tx at hub node. Consequently, APD array with a single lens was also implemented as a compact multi-Rx at hub node.

When the VCSEL beams (wavelength at $850 \mathrm{~nm}$ ) pass thru a $6-\mathrm{mm}$ focal length lens, the beams were uniformly expanded as shown in Fig. 3. Consequently, the VCSEL emitting cell positions or the beam service area (cell) positions were reversed both of column and row as shown in the upper part of Fig. 3. The average optical power of each VCSEL was approximately equal to $2 \mathrm{~mW}$. A 4-meter operating communication path with respected to center of $\mathrm{VC}$ SEL array was designed resulting $80-\mathrm{cm}$ diameter of service area of each VCSEL with 13.2-cm overlapped area or handover region. At 4 meter with respected to center of $\mathrm{VC}$ SEL array, optical power density was approximately equal to $0.40 \mu \mathrm{W} / \mathrm{cm}^{2}$ satisfying eye-safe operation. The middle far field infrared (IR) viewgraph of (cell) beam placement from $35 \mathrm{~cm}$ distance with respect to the center of hub node is shown in the lower part of Fig. 3. The overlapped area is for handover process when users move in/out of service area in each communication service cell. By using the same concept of OMC-Tx in the upper part of Fig. 3, the Rx (APD) array with a 6-mm focal length defocusing lens at hub node can recognize user terminal positions. For example, when user terminal located in service area A1, APD A1-cell can identify where user was. Hub node was also able to predict in advance where user could probably move, e.g. to A2-cell, B1-cell, B2-cell, or out of service area of this hub node. Thus, VCSEL array and APD array with lens (see Fig. 1) were required as compact $\mathrm{Tx}$ and $\mathrm{Rx}$ at hub node for OMC system, respectively.

At the user terminal, only a single VCSEL with a 3.5$\mathrm{mm}$ focal length lens placing $1.3 \mathrm{~mm}$ in front of VCSEL was required for signal transmission to hub node. However, position detection for moving user was also required, so a 4 channel APD with a 6-mm focal length lens placing $1.3 \mathrm{~mm}$ in front of APD surface was implemented as receiver at user terminal. Consequently, the 4-channel APD is simultaneously applicable for high speed communication and position detection. $\mathrm{D}_{3} \& \mathrm{D}_{4}$ axis and $\mathrm{D}_{1} \& \mathrm{D}_{2}$ axis are defined $x$ and $y$ axis, respectively, as shown in the upper part of Fig. 4 .

Sensitivity of user terminal Rx is $28.13 \mathrm{nW} /$ channel. The 4-channel APD implementing as user terminal Rx has the total sensitivity at $0.113 \mu \mathrm{W}$. At this operating optical

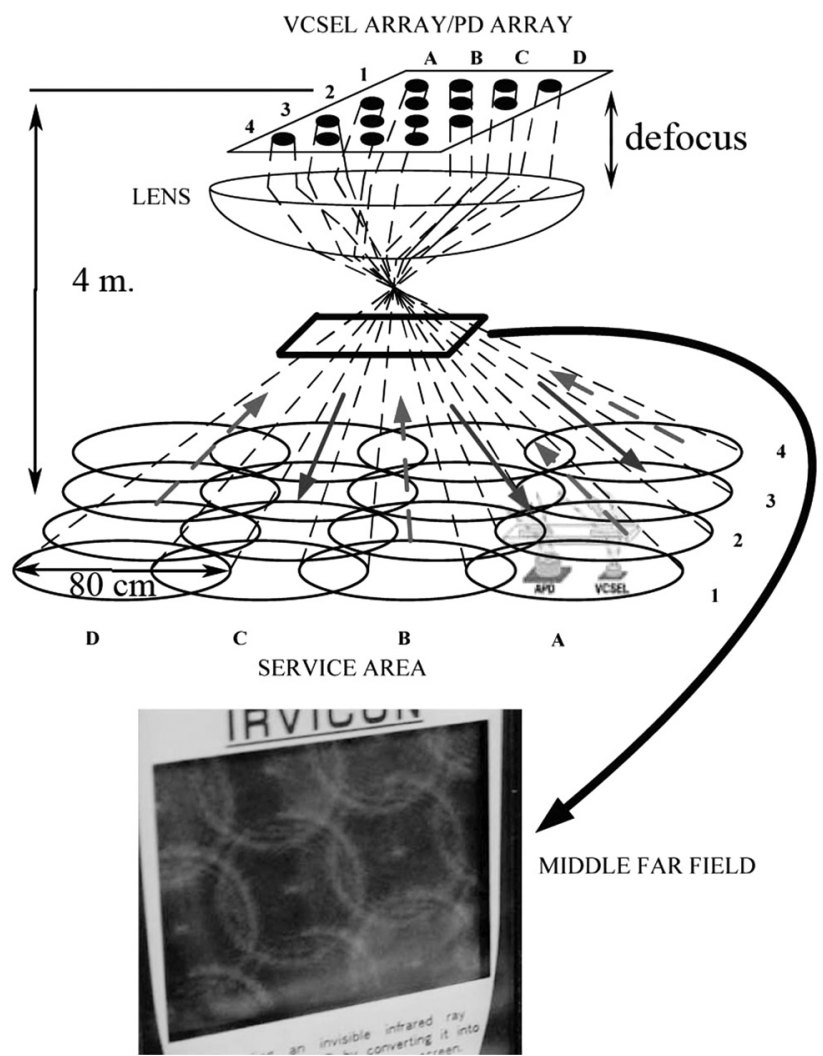

Fig. 3 The expansion of OMC beam service area and middle far field IR viewgraph of cell beam positioning of hub node at $35 \mathrm{~cm}$ distance.

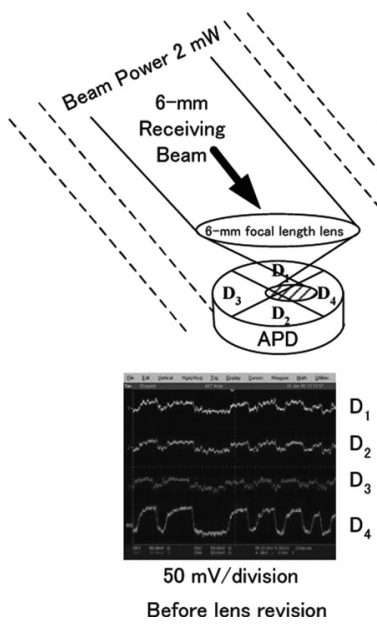

(a)
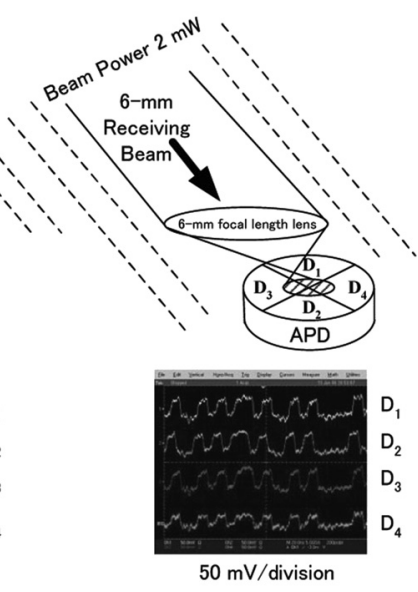

After lens revision

(b)
Fig. 4 (a) The misalignment of receiving APD at user terminal and its optical output intensity, (b) the revising lens position of receiving APD at user terminal and its optical output intensity.

signal level, signal-to-noise ration (SNR) was calculated [19] as 2.22 featuring from signal current $\left(\mathrm{i}_{s}\right)=1.44 \mu \mathrm{A}$, quantum noise current $\left(\mathrm{i}_{Q}\right)=0.34 \mu \mathrm{A}$, bulk dark current $\left(\mathrm{i}_{D B}\right)=0.13 \mu \mathrm{A}$, thermal noise current $\left(\mathrm{i}_{T}\right)=0.18 \mu \mathrm{A}$ and surface dark current is negligible due to avalanche gain; under condition of operating wavelength is $850 \mathrm{~nm}$, quantum efficiency (q.e.) is $75 \%$, operating frequency bandwidth 


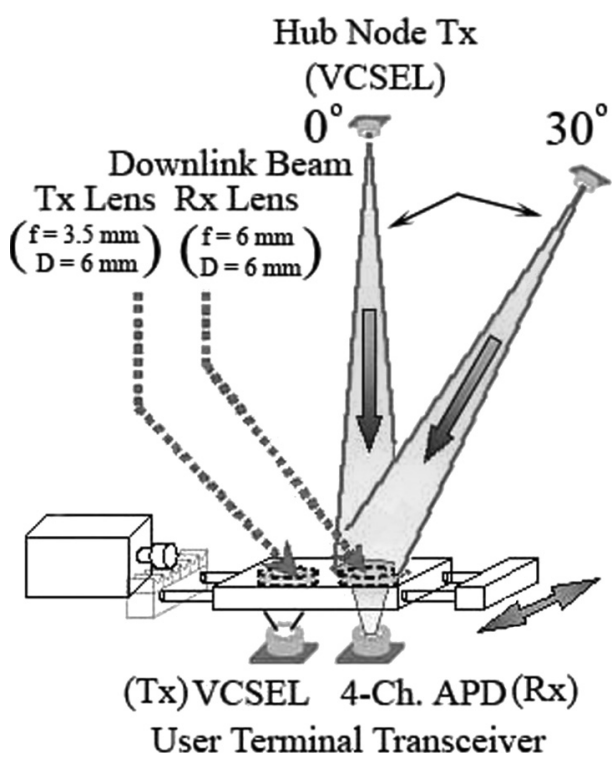

Fig. 5 The beam misalignment and posture revision experimental setup.

$(B W)$ is $100 \mathrm{MHz}$, load resistance $\left(R_{L}\right)$ is $50 \Omega$, operating temperature $(T)=20^{\circ} \mathrm{C}$ and avalanche multiplication $(M)$ is 100 . To improve the sensitivity of $\mathrm{Rx}$, the lens diameter change can improve the receiving sensitivity, even under eye-safe condition $\left(2 \mathrm{~mW} / \mathrm{cm}^{2}\right.$; IEC class I). However, the compactness of the user terminals was strongly required. On the other hand, the typical minimum received optical power is less than $-41 \mathrm{dBm}$ (approximately $80 \mathrm{nW}$ ) when $100 \mathrm{Mpbs}$ data rate at $850 \mathrm{~nm}$ wavelength collimating beam. Even the SNR is small, but SNR can be improved by the post amplifier to filter and amplify to correct the signal level and frequency band.

When user terminal moves to the edge or overlapped section of service area, the 4-channel APD with the 6-mm focal length lens results the misalignment. For example, when $\mathrm{D}_{3}$ detects reducing signal and $\mathrm{D}_{4}$ detects the increasing signal while $\mathrm{D}_{1}$ and $\mathrm{D}_{2}$ detect equally increasing or decreasing signal, it implies that hub node was now on the left hand side (close to $\mathrm{D}_{3}$, see Fig. 4(a)). The Rx was required to revise its posture to get balance signal of $D_{1}, D_{2}$, $\mathrm{D}_{3}$, and $\mathrm{D}_{4}$. If every channel was balanced, it implied that posture control was completed to receiving beam as shown in Fig. 4(b). Using 4-channel APD as Rx caused optical signal degradation because of the intensity of each cell of 4-channel APD was divided by 4 after posture revised, so high speed signal from 4-channel APD must be combined to overcome optical signal degradation problem before processing in data and control splitter module (see Fig. 2).

Ref.[10]-[13] showed the better solution using 5channel APD (one APD at the center surrounding with another 4 APDs) for signal detection but cost is high. However, the proposed 4-channel APD can detect all signals and can be summed 4-channel signals after amplification. This summation can reduce the random noise effect. Fig. 5 shows the beam misalignment and posture revision experimental

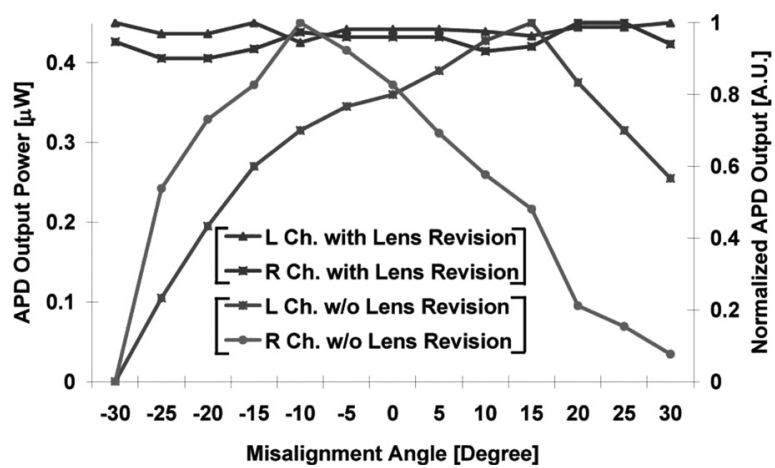

Fig. 6 Detected signal of left $\left(D_{3}\right)$ and right $\left(D_{4}\right)$ channels with and without posture control.

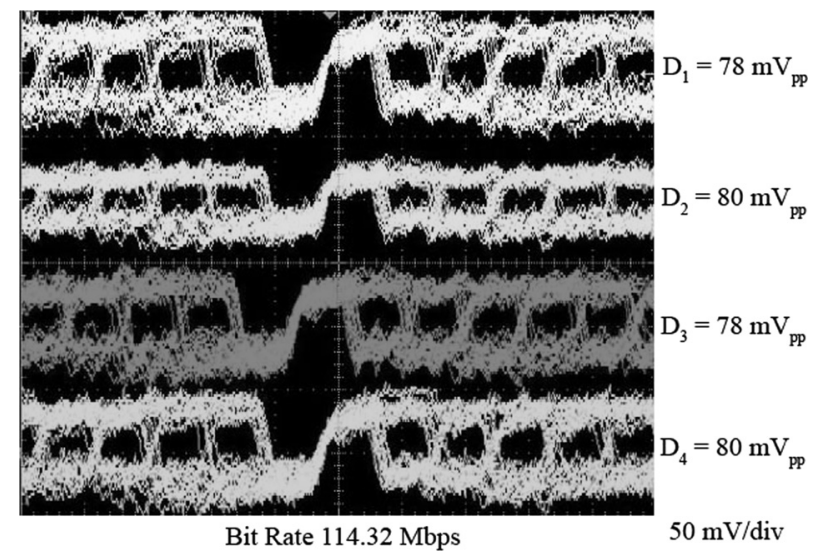

Fig. 7 Eye diagram of 114.32-Mbps received signals from 4-channel APD after posture revision.

setup. A VCSEL was implemented as a single cell of hub node VCSEL array with various angles (simulated VCSEL cell positions). The results of lens misalignment and its posture control at 1 meter distance with respect to the center of hub node are shown in Fig. 6 . The detecting angle is $60^{\circ}$ $\left( \pm 30^{\circ}\right.$ with respect to center of 4-channel APD) by moving lens distance only $2 \mathrm{~mm}$. From the experiment, posture control seemed to operate in wider angle but the focusing beam cannot be located on the APD surface equally. When posture revision cannot be balance revised, the detected optical signal on 4-channel APD consequently degraded. After lens was revised its posture, the result was satisfied for balanced optical signal in both of left and right channels. Figure 7 shows the eye diagram of 114.32-Mbps received signal from 4-channel APD after posture was realized. The result shows good eye diagram and balanced intensity for decision circuit.

\section{Signal Processing and Autonomous Posture Control Configuration}

The signal data rate of optical wireless communication system is able to reach $1 \mathrm{Gbps}$ for each user to overcome recent millimetre wave RF wireless access systems. On the other hand, sub-Mbps control command signal is sufficient 
for real-time cell switching and user terminal autonomous posture control. However, data and control command must be combined in a single frame communication for communication robustness and alignment mechanism. The combined signal does not only for robust alignment mechanism but also for handover control. The high speed data acts as sub carrier of the control command. The data signal bit rate of an optical wireless system is very high (approximately 1 Gbps or more). The beam cell control command signal speed is sufficiently high even bit rate is a few kbps.

In this experiment, approximately $100 \mathrm{Mbps}$ data signal and $9.6 \mathrm{kbps}$ control signal were combined by a largescale-integrated circuit (LSI) using a phase-locked loop frequency-shift keying (PLL-FSK) technique as frequency synthesizer (see Fig. 8(a)). The $100 \mathrm{Mbps}$ non return to zero (NRZ) data signal and the $9.6 \mathrm{kbps}$ control command signal were successfully combined by LSI using the PLL-FSK technique [14] and transmitted via VCSEL both of hub node and user terminal. At Rx of hub node and user terminal, clock recovery scheme successfully restored data signal and

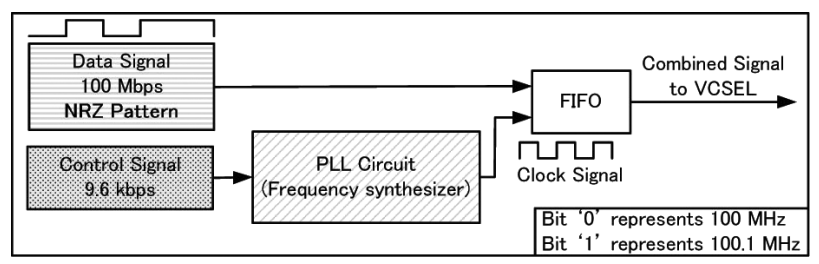

(a)

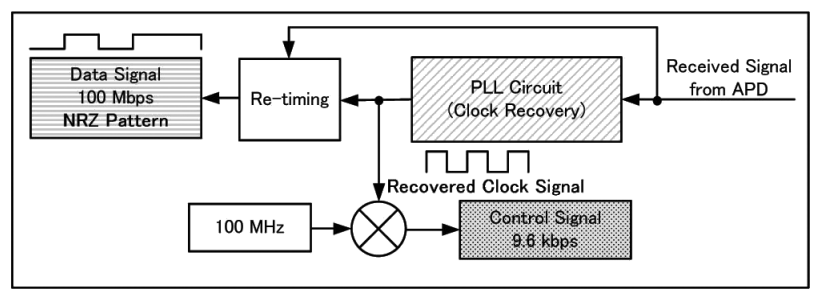

(b)

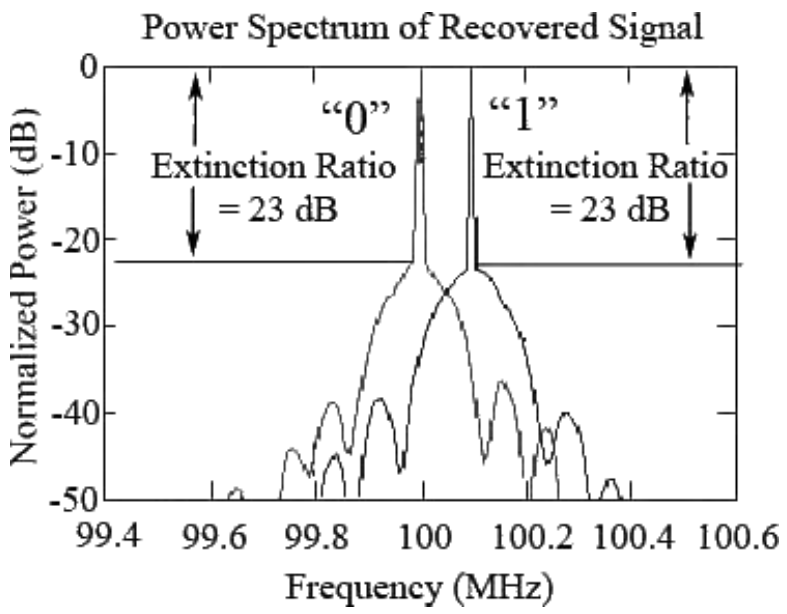

(c)

Fig. 8 Data and control signal combination scheme, (b) Data and control signal recovery scheme, (c) Clock recovered analyzed spectrum of received signal. control command signal as shown in Fig. 8(b) [14]. Both of signal combination and restoration were performed by $0.1 \%$ synthesized frequency modulation. Figure 8(c) shows the clock recovery spectrum of the received signal. The 100.1 $\mathrm{MHz}$ clock frequency showed the " 1 " keying data and the $100 \mathrm{MHz}$ frequency showed the " 0 " keying data of the control signal. The " 1 " keying and " 0 " keying frequencies were compared by PLL clock recovery circuit. The signal containing a high speed data and cell number information from the hub node was received by user terminal. The user terminal reported its position in the coverage area of the current cell and requests handover. The direction of laser beam of the hub node can be switched by cell switching module. Finally, beam direction and posture of each user terminal can refined without link break or re-initialization.

To initialize link, when user terminal moved into beam service area, the 4-channel APD received focused signal from hub node. This signal was filtered and amplified by S.C. (see Fig. 2), then transmitted to processing control (programmable integrated circuit: PIC) to revise lens posture by sending a driving command to actuator driver to control stepping motor for revising user terminal posture. PIC controller has internal 8-bit analog to digital converter (A/D). The quantization level of this $\mathrm{A} / \mathrm{D}$ is approximately $19.53 \mathrm{mV}$. To protect electronic noise fluctuation, so $10 \%$ differences in $D_{1}$ and $D_{2}$ or $D_{3}$ and $D_{4}$ were acceptable range for autonomous posture control. The algorithm for autonomous posture control is shown in Fig. 9. This algorithm is fast enough, due to a few millisecond loop processing, so there is no overshoot for posture control. The control mechanism is the autonomous posture sensing and user terminal control. Not only hub node beam direction control, but also user terminal posture sensing and control were important for a stable face-to-face link with a moving user. Down link optical signal within $\pm 30^{\circ}$ misalignment from the hub node can be detected. Then, user terminal posture and up link

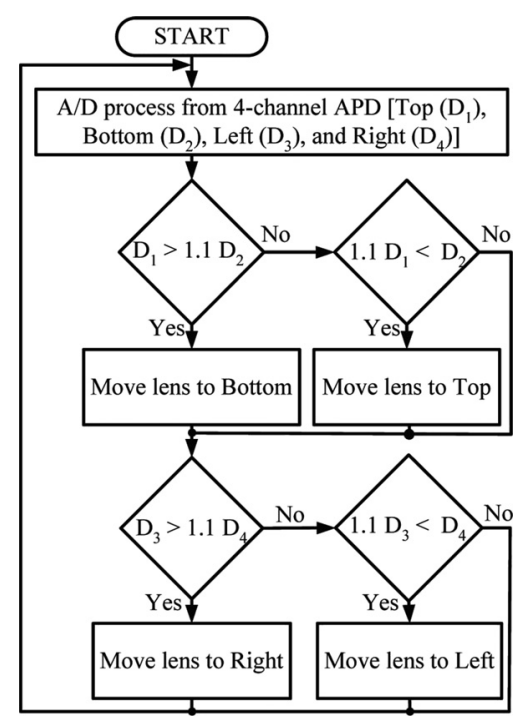

Fig. 9 Autonomous posture control algorithm. 


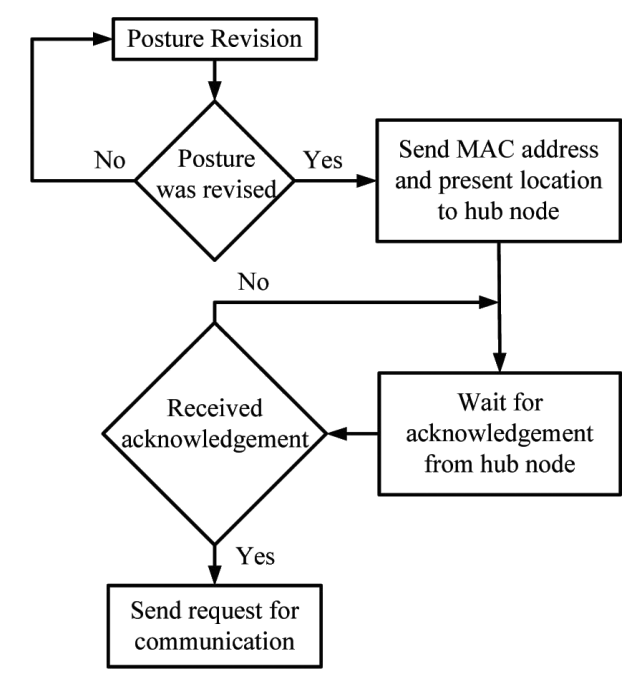

Fig. 10 Communication procedure after posture was revised.

VCSEL beam can be revised. After posture was completely realized, processing controller of user terminal transmitted a command to initialize link (request for IP address) by sending MAC address and present location to hub node. Hub node received the signal and acknowledges link communication to complete link request. For further communication, user terminal transmitted a requested command via VCSEL to hub node. Hub node received this request and then, acknowledged the requested communication. It is similar protocol as link initialization. Figure 10 shows communication procedure after posture was revised.

Handover and autonomous posture control concepts is shown in Fig. 11. For handover process, hub node consists of VCSEL array as optical transmitter array, APD array as optical receiver array, switch matrixes and a PIC processing controller. The selected beam of the hub node simultaneously transmitted communication data and current cell number information to user terminal. If the user terminal moved out of the previous cell service area, user terminal sensed the posture of the user terminal and transmitted the handover requirement to hub node. Hub node switched the emitting beam for the next cell (see Fig. 11). The signal for this user was switched by electronic switching matrix. The edges of two cells were slightly overlapped. When user terminal moved to the edge of the previous cell, the signal intensity of the selected receiver was less than the threshold level. The average and different intensities of the signal were measured and transmitted to the autonomous posture control circuit. The processing controller automatically decided and controlled user terminal's posture. User terminal required the switching data transmission VCSEL number to the hub node. After VCSEL was switched, the received optical signal intensity and quality were recovered over the threshold level to maintain a downlink communication. This process was successfully demonstrated. Smooth beam switching and 114.32 Mbps eye diagram improvement using the control command from user terminal were obtained as shown

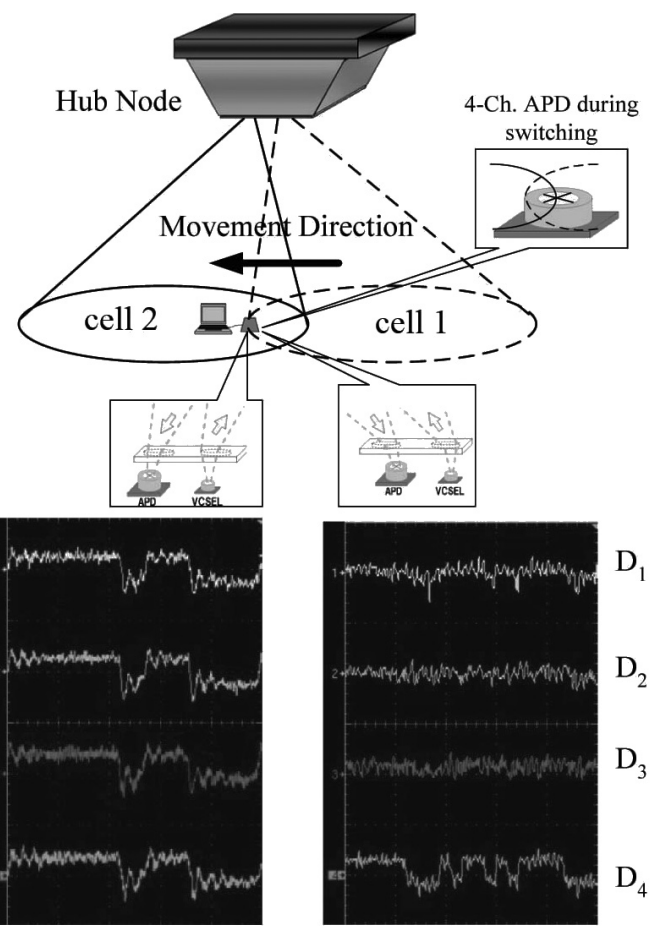

User Terminal After Switched User Terminal Before Switched

Fig. 11 Handover and autonomous posture control concepts.

in Fig. 11. When user terminal moved to the edge of a VCSEL cell service are of hub node, only one channel of user terminal Rx can detect optical signal from hub node. So user terminal sent the handover requirement to hub node to switch the present service area to the new service area. After service area was switched and user terminal posture was revised the balanced optical signal can be re-obtained.

This system was designed for multi-user point-to-point link. The cell size around $80 \mathrm{~cm}$ was designed for a single user. However, if 2 or more users access at the same service area, downlink and uplink should be shared using carrier sense multiple access with collision detection-time sharing system (CSMA/CD-TSS) protocol which is weaker security. For multi-user accessibility, there is a very low probability that two or more users operate in the same service area due to small size service area only $80 \mathrm{~cm}$ diameter. However, if only transceivers of two or more users are placed in the same service area, the communication signal can be extracted using MAC address to identify which user requests the communication.

\section{Conclusions}

In summary, OMC has potential to develop to multi-users Gbps communication, due to simple optical layout and the advanced integration in optoelectronics. Optical wireless communication and autonomous beam control for multimoving user terminals using OMC technique was proposed. The design, implementation, and downlink demonstration of $114-\mathrm{Mbps}$ autonomous beam control optical wireless com- 
munication system bases on an OMC technique were presented. High speed (100 or more Mbps bit rate) or very high speed (Gbps bit rate) data signal can be simultaneously inserted with control command (cell number and/or handover) into single beam communication using PLL with FSK modulation scheme. The simple posture control with OMC system is capable of considerable development to improve large capacity communication, over Gbps, because of optic is implemented as medium. It is a communication system with very high carrier frequency, owing to no radio regulation for bandwidth limit. In addition, it does not disturb the operation of electromagnetic sensitive devices. In order to obtain Gbps communication and flexible user number, all electronic module must be implemented in VLSI to reduce external noise, external capacitor, and to immune RF interference which consequently yields with compact OMC.

\section{Acknowledgments}

The authors would like to thank Hamamatsu Photonic Co., Ltd. for delivery 4-channel APDs. This work was partially supported by foundation of "High-Tech Research Center Development Program."

\section{References}

[1] IEEE Computer Society, "IEEE Standard $802.11 \mathrm{G}^{\mathrm{TM}}$," pp.14-16, 2003.

[2] M.J. McCullagh, P.P. Smyth, D.R. Wisely, and P.L. Eardley, "Optical wireless lans: Application and systems," IEE Colloquium on Cordless Computing - Systems and User Experience, pp.8/1-8/3, 1993

[3] R. Ramirez-Iniguez and R.J. Green, "Indoor optical wireless communications," IEE Colloquium on Optical Wireless Communications (Ref. no.1999/128), pp.14/1-14/7, 1999.

[4] N.A. Riza, "Reconfiguration optical wireless," Lasers and ElectroOptics Society 1999 12th Annual Meeting, LEOS'99, vol.1, pp.70 71, 1999.

[5] D. Wisely and I. Neild, "A $100 \mathrm{Mbit} / \mathrm{s}$ tracked optical wireless telepoint," Personal, Indoor and Mobile Radio Communications, vol.3, pp.964-968, 1997.

[6] K. Nishida, "A proposal of multi beam transmitter for non-directed diffuse indoor optical wireless communication system," Personal, Indoor and Mobile Radio Communications, vol.1, pp.242-246, 1996.

[7] K. Nonaka, M. Hamada, and Y. Isobe, "High speed optical wireless access with a smart beam control mechanism," APOC03, pp.449450, Wuhan, China, 2003.

[8] K. Nonaka and Y. Isobe, "High speed optical wireless access with VCSEL-array beam micro-cell system," ECOC 2004, pp.127-128, Stockholm, 2004.

[9] K. Nonaka, Y. Shima, A. Posri, and M. Tachibana, "Highspeed optical micro-cell wireless system for moving user access terminal with VCSELs and receivers array," LEOS Summer Topical Meetings, 2005, pp.37-38, San-Diego, 2005.

[10] C. Tangtrongbenchasil, Y. Hamada, T. Kato, T. Watanabe, and K. Nonaka, "Indoor, compact, and smart control high speed optical wireless communications," ITC-CSCC 2006, vol.2, pp.5-8, Chiangmai, Thailand, 2006

[11] D.C. O’Brien, G.E. Faulkner, K. Jim, E.B. Zyambo, D.J. Edwards, M. Whitehead, P. Stavrinou, G. Parry, J. Bellon, M.J. Sibley, V.A Lalithambika, V.M. Joyner, R.J. Samsudin, R. Atkinson, D.M. Holburn, and R.J. Mears, "High-speed integrated transceivers for optical wireless," IEEE Commun. Mag., vol.41, no.3, pp.58-62, 2003.

[12] D.C. O’Brien, G.E. Faulkner, K. Jim, E.B. Zyambo, D.J. Edwards, M. Whitehead, P. Stavrinou, G. Parry, J. Bellon, M.J. Sibley, V.A. Lalithambika, V.M. Joyner, R.J. Samsudin, R. Atkinson, D.M Holburn, and R.J. Mears, "Integrated transceivers for optical wireless communications,” IEEE J. Sel. Top. Quantum Electron., vol.11, no.1, pp.173-183, 2005.

[13] D.C. O’Brien, G.E. Faulkner, K. Jim, D.J. Edwards, E.B. Zyambo, P. Stavrinou, G. Parry, J. Bellon, M.J. Sibley, R.J. Samsudin, D.M Holburn, V.A. Lalithambika, V.M. Joyner, and R.J. Mears, "Experimental characterization of integrated optical wireless components," IEEE Photonics Technol. Lett., vol.18, no.8, pp.977-979, 2006.

[14] P. Ampornrat and M. Tachibana, "Design of transmitting and receiving section of optical wireless access using PLL," IQEC/CLEO-PR, 2005

[15] D.M. Kuchta, Y.H. Kwark, C. Schuster, C. Baks, C. Haymes, J. Schaub, P. Pepeljugoski, L. Shan, R. John, D. Kucharski, D. Rogers, M. Ritter, J. Jewell, L.A. Graham, K. Schrödinger, A. Schild, and H.-M. Rein, "120-Gb/s VCSEL-based parallel-optical interconnect and custom 120-Gb/s testing station," J. Lightwave Technol., vol.22, pp.2200-2212, 2004

[16] A.V. Krishnamoorthy, K.W. Goossen, L.M.F. Chirovsky, R.G Rozier, P. Chandramani, S.P. Hui, J. Lopata, J.A. Walker, and L.A. D'Asaro, " $16 \times 16$ VCSEL array flip-chip bonded to CMOS VLSI circuit," IEEE Photonics Technol. Lett., vol.12, no.8, pp.1073-1075, 2000

[17] H. Kosaka, "Smart integration and packaging of 2-D VCSEL's for high-speed parallel links," IEEE J. Sel. Top. Quantum Electron., vol.5, no.2, pp.184-192, 1999.

[18] K.J. Ebeling, "Applications of VCSELs for optical interconnects," 24th European Conference on Opt. Commun., vol.3, pp.27-31, 1998.

[19] G. Keiser, "Photodetectors," in Optical Fiber Communications, 3rd ed., pp.243-268, McGraw-Hill, Singapore, 2000.

[20] R.W. Waynant and M.N. Ediger, "Infrared detectors," in ElectroOptics Handbook, pp.17.1-17.25, McGraw-Hill, USA, 1993.

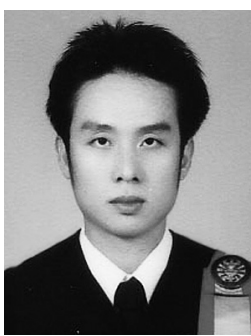

Charoen Tangtrongbenchasil was born in Bangkok, Thailand in 1979. He received the B.Eng. (Electrical Engineering) and M.Sc. (Engineer) degrees from Department of Electrical Engineering, Sirindhorn International Institute of Technology, Thammasat University, Thailand in 2000 and 2002, respectively. He is now pursuing Ph.D. at Department of Electronic and Photonic Systems Engineering, Kochi University of Technology, Kochi, Japan. His interested researches are optical communication, optical sensor, analog-digital signal processing, and autonomous system. He is a student member of IEEE. 


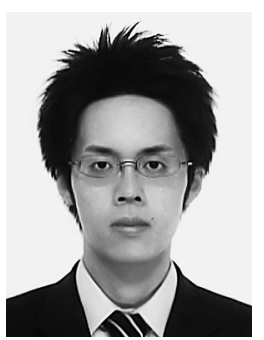

Yoichi Hamada was born in Kochi Prefecture, Japan in 1984. He entered at Department of Electronic and Photonic Systems Engineering, Kochi University of Technology, Kochi, Japan in 2003. He is currently studying optical communications. His interesting research is optical wireless communication.

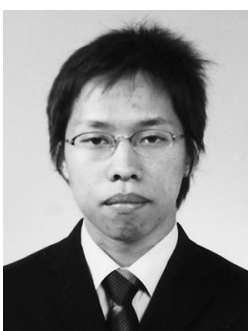

Toshihiro Kato was born in Kagawa Prefecture, Japan in 1985. He is now an undergraduate student in Nonaka laboratory, Department of Electronic and Photonic Systems Engineering, Kochi University of Technology, Kochi, Japan. His current research is the optical wireless communication based micro-cell system.

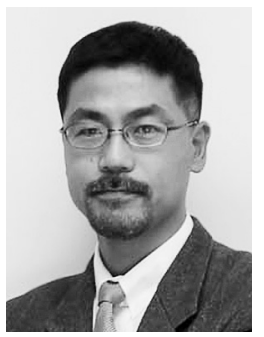

Koji Nonaka was born in Kumamoto Prefecture, Japan in 1961. He received B.E. and M.E. degrees in physics from Osaka University, Osaka, Japan in 1985 and 1987, respectively. $\mathrm{He}$ also received $\mathrm{Ph} . \mathrm{D}$. degree in engineering from Osaka University in 1997. In 1987, he joined the NTT Opto-electronics Laboratories. In 2000, he moved to Kochi University of Technology, Kochi, Japan. He is now a professor at Frontier Engineering Course, Kochi University of Technology. His interested researches are optical communications, optical signal processing and optic sensing based on functional laser diode. He is a member of the Japan Society of Applied Physics, Japan Society of Optics, and IEEE-LEOS. 